\title{
Artificial Intelligence in Cancer Staging: Limitless Potential or Passing Fad?
}

\author{
John W. Kunstman, MD, MHS ${ }^{1,2}$ \\ ${ }^{1}$ Department of Surgery, Section of Surgical Oncology, Yale University School of Medicine, New Haven, CT \\ ${ }^{2}$ VA Connecticut Health System, West Haven, CT
}

In this issue of Annals of Surgical Oncology, a distinguished group of international coauthors utilized a novel machine learning approach to modeling prognosis in patients with intrahepatic cholangiocarcinoma (ICC). ${ }^{1}$ Using a database spanning four continents, Tsilimigras and colleagues of the International Intrahepatic Cholangiocarcinoma Study Group found that tumor number, tumor size, albumin-bilirubin grade (ALBI), and preoperative lymph node involvement were most predictive of overall survival among patients undergoing resection for ICC. Of these findings, perhaps only ALBI will be unfamiliar to some practitioners; it is a metric for assessing liver function that was originally defined in patients with hepatocellular carcinoma but was recently shown to be prognostic in ICC as well by the current Study Group. ${ }^{2,3}$ Based on these four criteria, operative patients could be stratified four subgroups with excellent discrimination of overall survival.

It will come as no surprise to hepatobiliary surgeons that patients with ICC featuring large or multifocal tumors, regional lymphatic spread, or poor liver function will suffer comparatively poor survival next to their counterparts with more favorable disease characteristics. The staging of ICC, which was evaluated jointly with hepatocellular carcinoma until the 6th edition of the American Joint Committee on Cancer (AJCC) staging system, remains challenging and many have noted the shortcomings of the AJCC's current 8 th edition. ${ }^{4,5}$ In that context, this study makes a strong argument for altering the surgeon's approach to

(C) Society of Surgical Oncology 2020

First Received: 16 December 2019;

Published Online: 3 January 2020

J. W. Kunstman, MD, MHS

e-mail: john.kunstman@yale.edu management and more strongly weighing liver function in selecting patients for resection. For instance, patients with three or more tumors were found to have survival inferior to those with metastatic disease (AJCC stage 4) despite undergoing resection. Surgeons continue to debate whether multifocal ICC signifies metastatic or locoregional (satellitosis) disease, and these data strongly argue that resection offers minimal benefit to patients with multiple tumors except in very highly selected cases. As more data accumulates, the iterative refinement of ICC staging and selection for surgery will surely continue to improve.

Certainly, a deeper analysis of the prognostic features of ICC is both noteworthy and valuable to surgeons. However, the most significant contribution of the manuscript by Tsilimigras et al. is its methodology. As bigger and more inclusive datasets are amassed in many areas of medicine (nicely illustrated by the international database analyzed here), investigators must search for new ways to transform these resources into usable information. ${ }^{6,7}$ Broadly speaking, machine learning techniques, such as the Classification And Regression Tree (CART) predictive modeling employed here, offer an attractive way to analyze large datasets to determine patterns and trends. CART modeling was first described in 1984 by Breiman and is one of the most useful applications of machine learning. ${ }^{8}$ In CART analysis, patterns are determined computationally via inference, mimicking the human process of learning by example. ${ }^{9,10}$ Thus far, applications have been wide-ranging but with a particular focus on clinical prediction and prognostic models. ${ }^{11,12}$ Logistic regression, the traditional modeling technique for these applications, remains a key approach but relies on known rules and variables fixed by the investigator. ${ }^{13}$ Machine learning, when unsupervised, allows significant output variables to be deduced in an unbiased fashion, possibly revealing previously unknown 
patterns or findings. Savvy human investigators remain crucial to the process in order to determine which of these findings are not only statistically significant but have real clinical relevance.

Tsilimigras et al. illustrate both the great promise and potential drawbacks of using machine learning in analyzing surgical datasets. As the availability of ever-larger datasets continues to improve, the quality of the input data continues to be the limiting factor in attempts to answer practice-changing questions. For ICC, these questions include not only candidacy for surgery as examined here but also selection for neoadjuvant or adjuvant systemic therapy, best surgical approach, the role of transplantation, and many others. Addressing these questions requires large datasets of greatly increased granularity. Other applications of artificial intelligence, such as natural language processing of electronic medical records, hold promise in addressing these shortcomings. Conventional statistical approaches, such as logistic regression, will continue to be important, but machine learning will prove to be a major addition to the investigator's toolkit, especially in datasets vulnerable to bias. In the future, the opening efforts of Tsilimigras and colleagues will prove to be prescient as efforts to refine surgical decision-making are augmented by this powerful new approach.

\section{REFERENCES}

1. Tsilimigras DI, Mehta R, Moris D, et al. A machine-based approach to preoperatively identify patients with the most and least benefit associated with resection for intrahepatic cholangiocarcinoma: an international multi-institutional analysis of 1146 patients. Ann Surg Oncol. 2019. https://doi.org/10.1245/ s10434-019-08067-3.

2. Johnson PJ, Berhane S, Kagebayashi C, et al. Assessment of liver function in patients with hepatocellular carcinoma: a new evidence-based approach-the ALBI grade. J Clin Oncol. 2015;33(6):550-8.
3. Tsilimigras DI, Hyer JM, Moris D, et al. Prognostic utility of albumin-bilirubin grade for short- and long-term outcomes following hepatic resection for intrahepatic cholangiocarcinoma: a multi-institutional analysis of 706 patients. J Surg Oncol. 2019;120(2):206-13.

4. Spolverato G, Bagante F, Weiss M, et al. Comparative performances of the 7th and the 8th editions of the American Joint Committee on Cancer staging systems for intrahepatic cholangiocarcinoma. J Surg Oncol. 2017;115(6):696-703.

5. Sasaki K, Margonis GA, Andreatos N, et al. Serum tumor markers enhance the predictive power of the AJCC and LCSGJ staging systems in resectable intrahepatic cholangiocarcinoma. HPB (Oxford). 2018;20(10):956-65.

6. Miller DD, Brown EW. Artificial intelligence in medical practice: the question to the answer? Am J Med. Feb 2018;131(2):129-33.

7. Hashimoto DA, Rosman G, Rus D, Meireles OR. Artificial intelligence in surgery: promises and perils. Ann Surg. 2018;268(1):70-6.

8. Breiman L. Classification and regression trees. Belmont: Wadsworth International Group; 1984.

9. Nunn ME, Fan J, Su X, Levine RA, Lee HJ, McGuire MK. Development of prognostic indicators using classification and regression trees for survival. Periodontol 2000. 2012;58(1):134-42.

10. Che D, Liu Q, Rasheed K, Tao X. Decision tree and ensemble learning algorithms with their applications in bioinformatics. $A d v$ Exp Med Biol. 2011;696:191-9.

11. Merath K, Hyer JM, Mehta R, et al. Use of machine learning for prediction of patient risk of postoperative complications after liver, pancreatic, and colorectal surgery. J Gastrointest Surg. 2019. https://doi.org/10.1007/s11605-019-04338-2.

12. Loftus TJ, Tighe PJ, Filiberto AC, et al. Artificial intelligence and surgical decision-making. JAMA Surg. 2019. https://doi.org/10. 1001/jamasurg.2019.4917.

13. Christodoulou E, Ma J, Collins GS, Steyerberg EW, Verbakel JY, Van Calster B. A systematic review shows no performance benefit of machine learning over logistic regression for clinical prediction models. J Clin Epidemiol. 2019;110:12-22.

Publisher's Note Springer Nature remains neutral with regard to jurisdictional claims in published maps and institutional affiliations. 